\title{
Metal Ion Implanted Compliant Electrodes in Dielectric Electroactive Polymer (EAP) Membranes
}

\author{
P. Dubois ${ }^{1, a}$, S. Rosset ${ }^{1, b}$, M. Niklaus ${ }^{1, c}$ M. Dadras ${ }^{2, d}$, and H. Shea ${ }^{1, e}$ \\ ${ }^{1}$ LMTS, EPFL, r. Jaquet-Droz 1, 2002 Neuchâtel, Switzerland \\ ${ }^{2}$ IMT, University of Neuchâtel, r. Jaquet-Droz 1, 2002 Neuchâtel, Switzerland \\ aphilippe.dubois@unine.ch, bsamuel.rosset@epfl.ch, ${ }^{\mathrm{c}}$ muhamed.niklaus@epfl.ch, \\ 'massoud.dadras@unine.ch, ${ }^{\mathrm{e}}$ herbert.shea@epfl.ch
}

Keywords: dielectric electroactive polymer actuators, EAP, DEAP, ion implantation, FCVA, PBII, PDMS

\begin{abstract}
One of the key factors to obtain large displacements and high efficiency with dielectric electroactive polymer (DEAPs) actuators is to have compliant electrodes. Attempts to scale DEAPs down to the $\mathrm{mm}$ or micrometer range have encountered major difficulties, mostly due to the challenge of micropatterning sufficiently compliant electrodes. Simply evaporating or sputtering thin metallic films on elastomer membranes produces DEAPs whose stiffness is dominated by the metallic film.

Low energy metal ion implantation for fabricating compliant electrodes in DEAPs presents several advantages: a) it is clean to work with, b) it does not add thick passive layers, and c) it can be easily patterned. We use this technology to fabricate DEAPs micro-actuators whose relative displacement is the same as for macro-scale DEAPs. With transmission electron microscope (TEM) we observed the formation of metallic clusters within the elastomer (PDMS) matrix, forming a nano-composite. We focus our studies on relating the properties of this nano-composite to the implantation parameters. We identified the optimal implantation parameters for which an implanted electrode presents an exceptional combination of high electrical conductivity and low compliance.
\end{abstract}

\section{Introduction}

Dielectric electroactive polymer (DEAP) actuators are an emerging class of actuators that have demonstrated high efficiency and large displacement (10\% to $100 \%$ elongation) for macro-scale ( $\mathrm{cm}$ to $\mathrm{m}$ ) devices, often referred to as artificial muscles [1]. Dielectric EAP are basically an elastomeric polymer sheet sandwiched between two compliant electrodes. Applying a voltage between the electrodes creates a compressive electrostatic force which squeezes the film and, under free boundary conditions, causes the film's area to expand (Fig. 1).

One of the key factors to obtain large displacements and high efficiency with dielectric electroactive polymer (DEAPs) actuators is to have compliant electrodes. Most macrosize DEAs have electrodes made of conducting grease or powder (metal or graphite) [3]. These techniques work well for devices whose area is greater than $1 \mathrm{~cm}^{2}$, but are difficult to master when one wants to pattern $\mu \mathrm{m}$ - to mm-size electrodes. Attempts to scale DEAPs down to the $\mathrm{mm}$ or $\mu \mathrm{m}$ range have encountered major difficulties, mostly due to the challenge of micropatterning sufficiently compliant electrodes [4]. Due to the 3 to 4 orders of magnitude larger Young's modulus of metals compared to elastomers, simply evaporating or sputtering thin $(\sim 100 \mathrm{~nm})$ metallic films on elastomer membranes produces electrodes whose stiffness is dominated by the metallic film. 
Low energy metal ion implantation for fabricating compliant electrodes in DEAPs presents several advantages compared to powder-based methods: a) it has a low stiffening effect on the membrane, b) it does not add thick passive layers, c) it has a limited impact on the PDMS optical transmission properties, d) it is clean to work with, and e) electrodes area can be easily patterned by shadow masking. We use this technology to fabricate DEAPs micro-actuators whose relative displacement is the same as for macro-scale DEAPs [5],[6]. The implantation process creates a nano-composite a few $\mathrm{nm}$ thick, located in the top tens of nm of the surface of the elastomer [7],[8]. The implanted dose must be high enough to obtain a conductive layer, i.e. by percolation between nanoparticles, but low enough not to reach the mechanical properties of a metallic film.
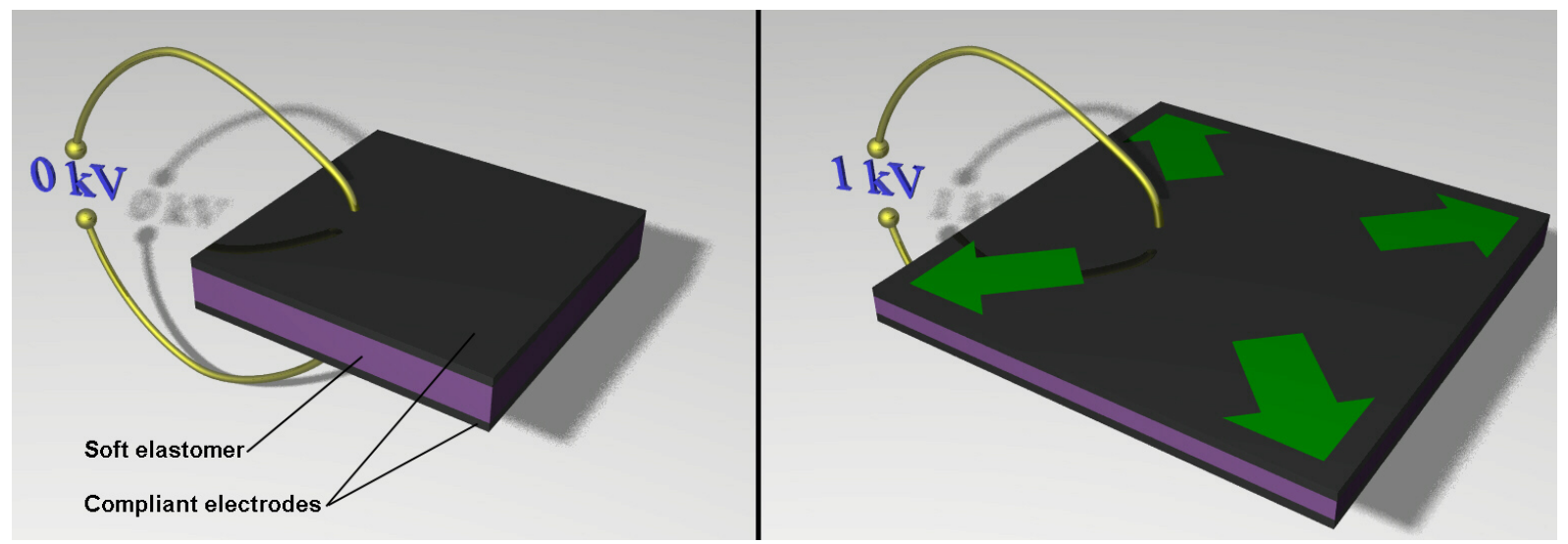

Fig. 1: Dielectric EAP (DEAP) principle [2]. When a voltage is applied to the electrodes (typically up to $1 \mathrm{kV}$ ), the electrostatic pressure squeezes the elastomer dielectric (right side). The volume of the dielectric being quasi constant, the whole structure stretches (in the case of free boundary conditions).

Most of the research on elastomers for macro-scale DEAs was conducted on three groups of elastomers based on: silicone, acrylic or polyurethane [1]. The main criteria for choosing an elastomer are it's Young's modulus, breakdown voltage, and compatibility with a specific fabrication process. We choose PDMS (Nusil CF19-2186 and Sylgard 186) for fabricating tests samples essentially for the following reasons: not required to pre-stretch the material and can be easily bonded (plasma $\mathrm{O} 2$ ) to micromachined silicon chips.

\section{Implantation Techniques}

Metallic ion implantation into polymers has been studied during the last decade, mostly to increase surface hardness or tune surface properties [9]-[12]. This method has been applied to different kinds of polymers, but little attention has been paid to elastomers. Most of the research has focused on energies readily achievable with standard commercial implanters, i.e., $30 \mathrm{keV}$ and above. 


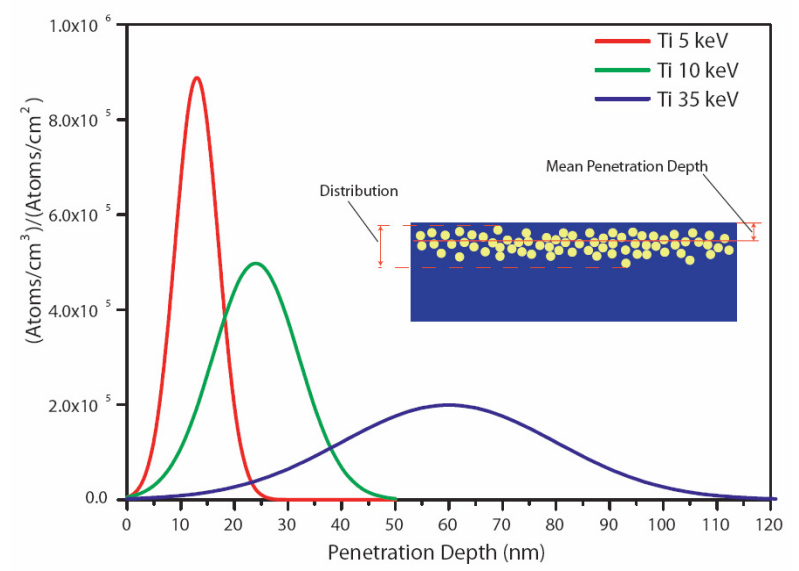

Fig. 2 SRIM simulation of Ti Ion penetration depth versus ion energy [13],[14]. Implanting at low energy allows obtaining a dense distribution of metallic particles close to the surface.

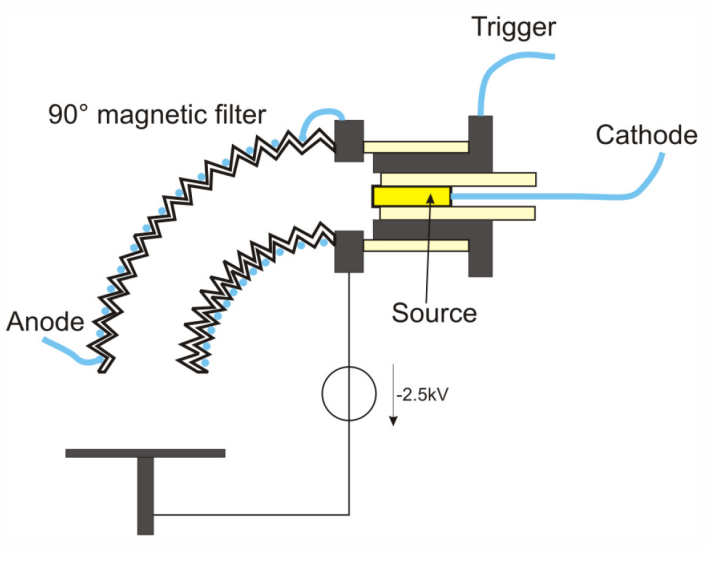

Fig. 3 Schematic of the FCVA implanter used for fabricating the tested devices. Metal ions are created by pulsed cathodic arcs and are then magnetically deflected in a $90^{\circ}$ bend filter. The substrate holder is polarized at a negative potential of a few $\mathrm{kV}$ to accelerate the ions [2].

For the low surface resistivity required by DEAP applications, the electrical conduction is dominated by percolation, i.e., conductive metal nano-particles entering into contacts, and therefore conductivity is highly dependent on the metal ion concentration in the elastomer. Monte Carlo simulations (Fig. 2) reveal that increasing the implantation energy broadens the ions distribution reducing their concentration.

Classical implanters used in the semiconductor industry have very low flux at low energies and generally only produce beams with energies above $10 \mathrm{keV}$. Implanting machines with higher flux at low energies are generally plasma-based. Among these plasma-based implantation machines, one can distinguish between machines generating a plasma flux (Filtered cathodic vacuum arc, FCVA) and those immersing the sample in the plasma (MEVVA, PBII, etc). FCVA machines present the advantage of filtering the ions and rejecting clusters for better implantation homogeneity. All plasma-based implantation machines can work in two modes, either with or without an acceleration potential on the sample. With an acceleration potential of a few $\mathrm{kV}$ on the sample, the electric field accelerates the ions that then penetrate a few nm into the sample. Due to the low heat conductivity of polymers (for instance $0.22 \mathrm{WK}^{-1} \mathrm{~m}^{-1}$ for the Polydimethylsiloxane model Sylgard 186), thin Polydimethylsiloxane (PDMS) membranes can burn if the implantation process generates too much heat as can occur at high ion fluence [6]. The plasma can be operated in a pulsed manner to reduce charging and heating of the sample.

We find filtered Cathodic Vacuum Arc (FCVA) to be the preferred implantation technique for creating conductive electrodes in elastomers, as it can produce large ions currents for different ion species ( $\mathrm{Ti}, \mathrm{Au}$ and Pd routinely used in our lab) at low energy, which allows implanting metallic particles at surface densities above $10^{16} \mathrm{at} / \mathrm{cm}^{2}$ a few nanometers below the surface, and in a short amount of time $(2-5 \mathrm{~min})$. The resulting electrodes exhibit low surface resistivity (0.1 to 10 $\mathrm{k} \Omega$ /square). In the FCVA system we used (based on RHK Arc 20 system, Fig. 3), $600 \mu$ s pulses were created at a frequency of $2 \mathrm{~Hz}$. Implantation rate is relatively high and doses of $10^{16} \mathrm{at} / \mathrm{cm}^{2}$ were obtained after 170 pulses [6].

We built our custom experimental FCVA setup, with a $1 \mathrm{~cm}^{2}$ beam, which is coupled to a $\mathrm{x}-\mathrm{y}$ stage for larger area scanning. Some commercial FCVA implanters, mainly designed and optimized to 
deposit hard coatings on cutting tools, do exist and could be applicable to treat large PDMS areas, provided their ion current can be set low enough that it won't burn the target and that the desired dose can be easily controlled.

\section{Macroscopic Characterization of Ion Implanted DEAPs Thin Film}

When implanting gold ions with an FCVA pulsed implanter on one side of a 20- $\mu$ m-thick PDMS membrane, we have found an optimum dose at $1.5 \cdot 10^{16} \mathrm{at} / \mathrm{cm}^{2}$, for which the resistivity drops to about $1 \mathrm{k} \Omega$ /square while the Young's modulus of the DEAP sandwich is increased by a about a factor 2 (Fig. 4) [8]. Gold electrodes were found to be more stable over time than titanium electrodes that present the drawback of oxidizing in air.

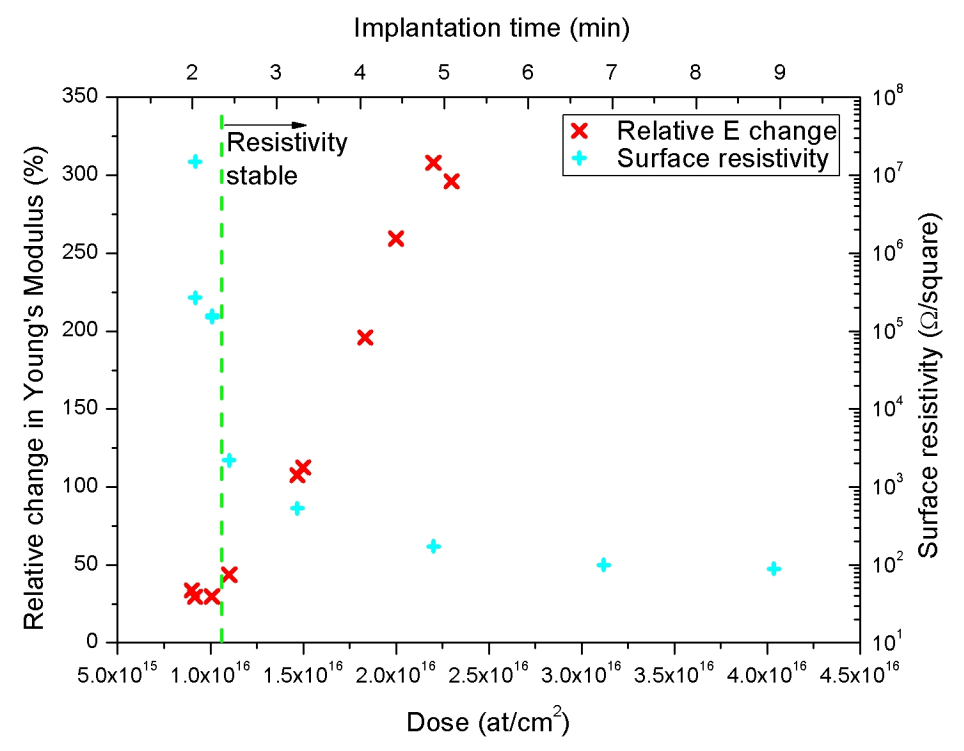

Fig. 4 Plot of the stiffening of membranes, as well as surface resistivity obtained by FCVA implantation into PDMS (Sylgard 186) of Gold for doses between $1 \cdot 10^{16} \mathrm{at} / \mathrm{cm}^{2}$ and $4 \cdot 10^{16} \mathrm{at} / \mathrm{cm}^{2}$ [8].

One of the important characteristics that a compliant electrode must exhibit is its ability to remain conductive during stretching (Fig. 5). Uniaxial tension tests were conducted on gold implanted samples that consisted of PDMS (Sylgard 186) stripes $(3 \mathrm{~mm}$ long $\times 8.5-18 \mathrm{~mm}$ wide $\times 200 \mu \mathrm{m}$ thick). Step measurements show that resistivity remains stable while holding a position and that deformation up to $40 \%-45 \%$ can be achieved with stable conductivity [8]. Resistivity increases from an initial value of $20-30 \Omega$, up to a few $\mathrm{k} \Omega$ at $45 \%$ strains, and becomes an open circuit for strains larger than 55\%. Samples implanted with classical implanters at $10 \mathrm{keV}$ and above required larger doses to achieve surface conductivity below $10 \mathrm{k} \Omega /$ square than those implanted with FCVA at $5 \mathrm{keV}$ [7].

For comparison, samples with an electrode made by Au cathodic sputtering lose their conductivity between 2 to $4 \%$ strain. Cyclic measurements confirm that there is no hysteresis within a cycle, and that after several tension-relaxation cycles, the resistivity of the undeformed stripe is not altered (Fig. 6). 


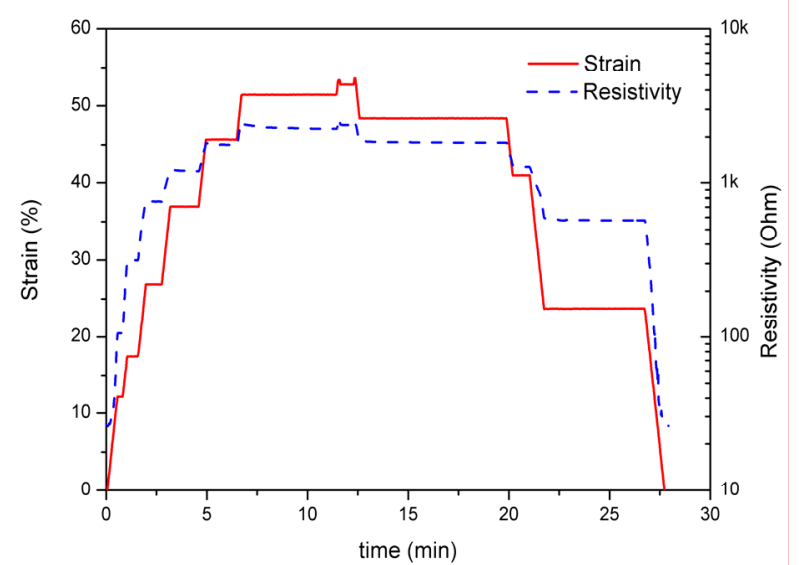

Fig. 5 Step measurement of electrical resistance and uniaxial strains between 0 and $55 \%$ of a PDMS stripe $\left(l_{0}=8.5 \mathrm{~mm} \mathrm{w}=3 \mathrm{~mm}\right)$ with an Au-implanted layer [8].

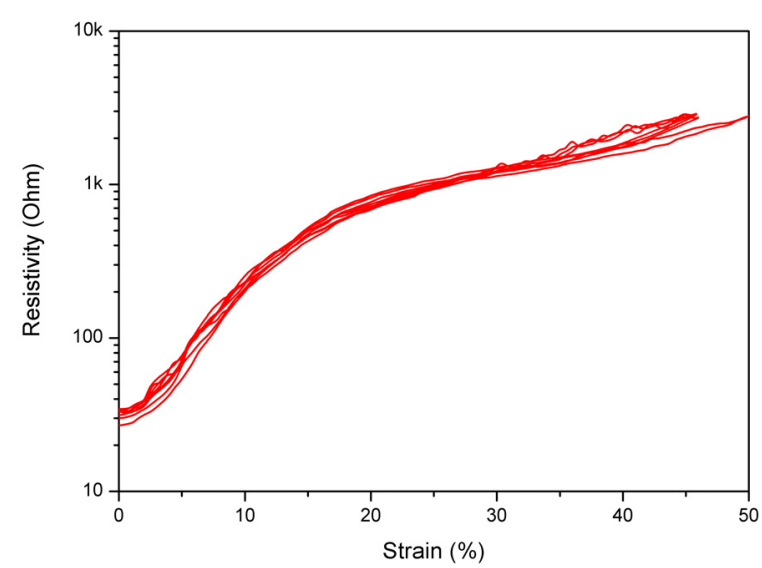

Fig. 6 Cyclic strain-resistance measurement of a gold-implanted PDMS stripe $\left(l_{0}=8.5 \mathrm{~mm} \mathrm{w}=3\right.$ $\mathrm{mm})[8]$.

Systematic measurements of DEAP thin films required the development of reproducible sample preparation techniques. We have focused our studies on the characterization of diaphragm actuators consisting in DEAP membranes fixed on top of rigid orifices patterned in bulk silicon chips (Fig. 7).

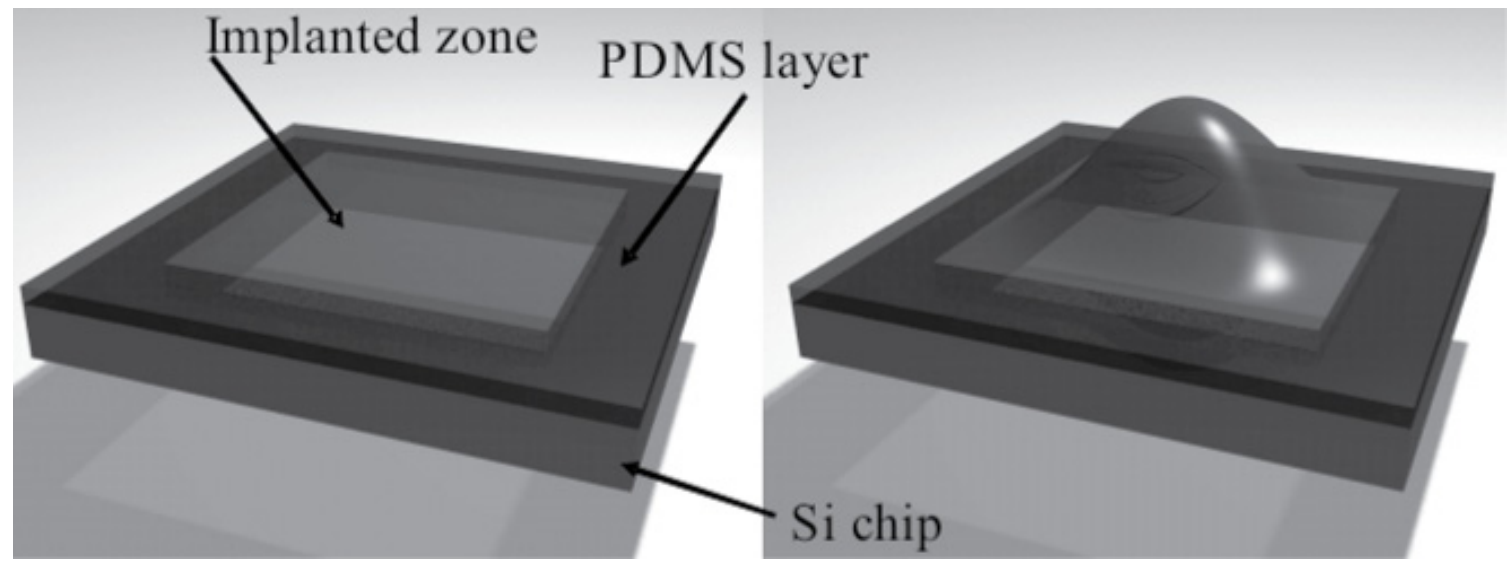

Fig. 7: Diaphragm actuators fabricated for characterization purposes [13]. They consist of an ion implanted PDMS membrane bonded on top of an orifice made in a silicon chip. Applying a voltage to the electrodes creates an electrostatic pressure which squeezes the elastomer dielectric and causes the membrane to buckle (right side).

We use a bulge test setup where we can apply a well defined gas differential pressure on the membrane and record its displacement with an optical profilometer [6]. With this setup we can characterize the mechanical properties of the membrane, i.e. Young's modulus and tensile stress, and quantify the impact of ion implantation.

By combining electrical actuation with an applied gas pressure we were able to measure the force displacement characteristics of the membrane [2]. This customized bulge test setup is an ideal tool for systematic DEAP membrane characterization. For a 3-mm-diameter 22.5 - $\mu \mathrm{m}$-thick clamped gold ion implanted DEAP membrane (PDMS Nusil CF19-2186, $1 \cdot 10^{16}$ at $/ \mathrm{cm}^{2}$ ) [2], we observed close to the dielectric breakdown limit $(800 \mathrm{~V}$ or $35.6 \mathrm{~V} / \mu \mathrm{m})$ a displacement of $200 \mu \mathrm{m}$ which 
represents $6.7 \%$ of the actuator's diameter. For the same actuator loaded with a distributed force of $7 \mathrm{mN}$, the electrically induced displacement was reduced to $86 \mu \mathrm{m}(2.87 \%$ of the actuator's diameter), leading to a mechanical work of $320 \mathrm{~nJ}$ (Fig. 9). The compliance of the electrodes was found to be crucial. Varying the implantation dose from $1 \cdot 10^{16}$ to $2 \cdot 10^{16} \mathrm{at} / \mathrm{cm}^{2}$ reduces by a factor 8 the mechanical work.

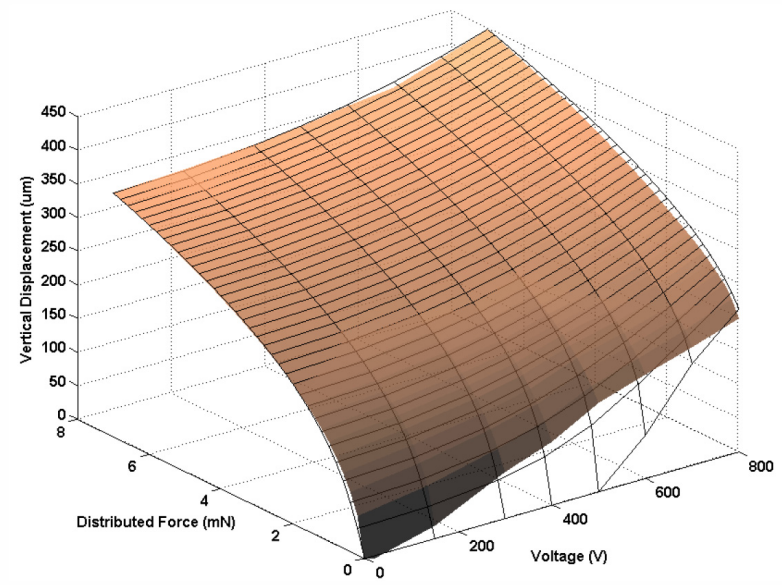

Fig. 8 Vertical displacement of the center of the membrane for voltages between 0 and $800 \mathrm{~V}$, and applied distributed force between 0 and $7 \mathrm{mN}(0-$ $990 \mathrm{~Pa})$ [2]. Wireframe: Theoretical model. Brown plane: datapoints.

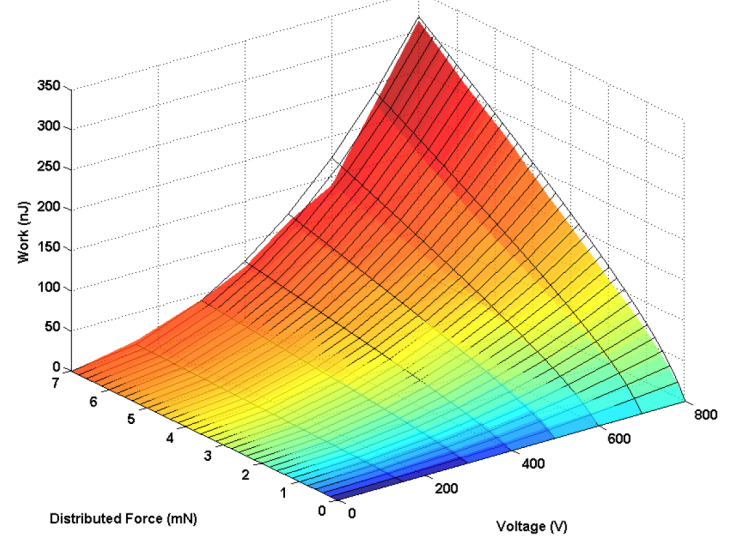

Fig. 9 Mechanical work as a function of applied voltage and distributed force [2]. Wireframe: Theoretical model. Color plane: datapoints.

Measurements show that ion metal implantation does not alter the dielectric strength of the PDMS, at least not for the low energies used here $(5 \mathrm{keV})$ [8]. Ion implantation can therefore be used to make compliant electrodes for DEAs without fear of altering the elastomer's dielectric strength.

\section{Microscopic Properties of Implanted Electrodes}

When implanting in an insulating matrix at high doses and low energy, metal ions stop a few $\mathrm{nm}$ below the surface, congregating together to form clusters that dominate the conduction mechanism. The depth of the layer depends on the implant energy, and the particle density and size distribution depend on the dose, as well as on diffusion, and hence the temperature of deposition and ion mobility. The thickness of the layers varies according to the ion type and implantation condition, and can be readily computed for low doses with the Monte Carlo simulation program SRIM 2003. The total thickness of this nanocomposite is usually below $100 \mathrm{~nm}$ (energy and material dependent, Fig. 2).

The conduction phenomena relate essentially to the proximity between electrically conducting clusters. Parameters such as average cluster size and cluster concentration have a strong influence on the conduction. The threshold conditions at which the implanted layer becomes highly conductive is the percolation level. A transmission electron microscope (TEM) image taken on a sample implanted at a moderate dose reveals the cluster arrangement just below the PDMS surface (Fig. 10). As seen on Fig. 11, higher doses result in implanted layers that are more compact, have lower resistivities and are stiffer. 


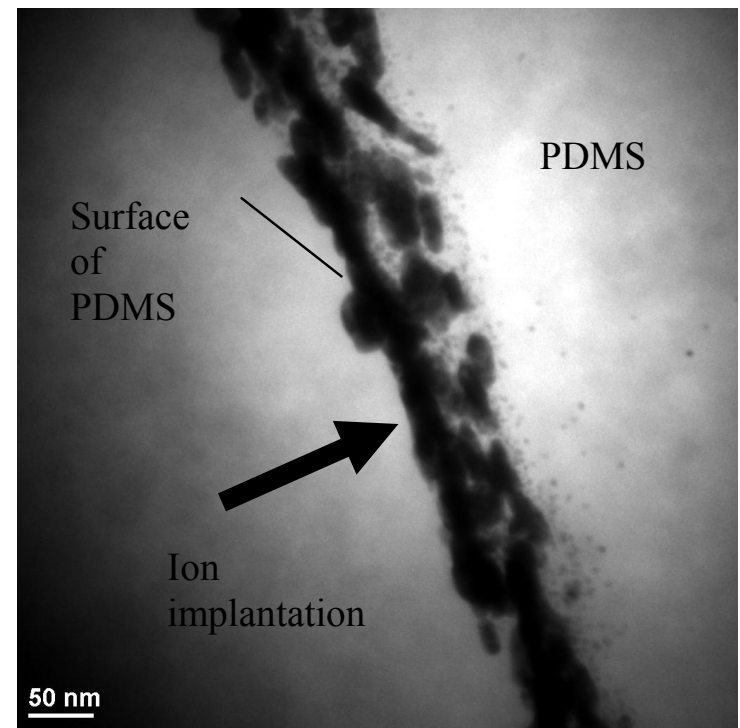

Fig. 10 TEM cross-section micrograph of a PDMS sample implanted at a dose of $1.5 \cdot 10^{16}$ at $/ \mathrm{cm}^{2}$.

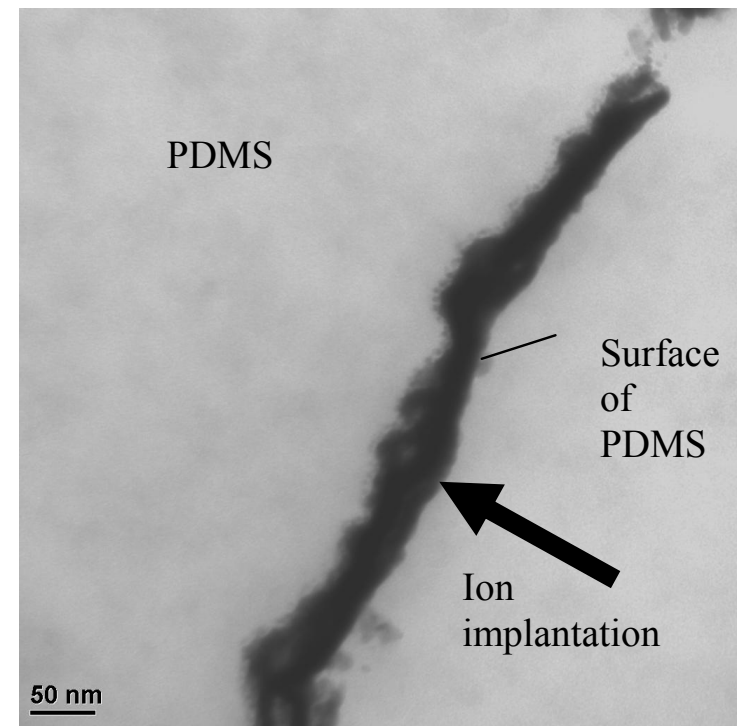

Fig. 11 TEM cross-section micrograph of a PDMS sample implanted at a dose of $2.3 \cdot 10^{16}$ $\mathrm{at} / \mathrm{cm}^{2}$.

\section{Conclusion and Outlook}

Ion implantation is an elegant technique to create electrodes on DEAP membranes combining exceptional characteristics that would be difficult to achieve with conventional approaches. To meet the requirements of high compliance and conductivity, ion implantation in PDMS has to be conducted at low energy. Filtered Cathodic Vacuum Arc (FCVA) implanters are better suited than conventional implanters that do not provide high fluence at low energy. For gold ions implanted into PDMS, the electrical resistivity drops by 5 orders of magnitude when the implanted dose is increased from $1 \cdot 10^{16}$ to $2 \cdot 10^{16}$ at $/ \mathrm{cm}^{2}$, which indicates the crossing of the percolation threshold.

Millimeter scale DEAPs actuator having electrodes fabricated by FCVA ion implantation exhibit excellent properties similar to macro scale devices. Gold implanted samples were found to have a stable resistivity of a few tens of Ohms at rest that increases up to a few $\mathrm{kOhm}$ when the sample reaches a strain of about $50 \%$. We observed typical cluster sizes of 2 to $20 \mathrm{~nm}$ that increases with increasing doses, and correlates with a stiffening of the membrane. The implantation dose must be properly chosen to enable a DEAP actuator achieve high output mechanical work, i.e. typically $1.5 \cdot 10^{16}$ at $/ \mathrm{cm}^{2}$ at $5 \mathrm{keV}$ implanting energy.

This post-polymerization electrode fabrication technique will benefit from further fundamental material studies of the nanocomposite formed by ion implantation and specific DEAP actuator developments. The best-suited implanters for this application, FCVA implanters, will have to be optimized to obtain consistent results on large surfaces without requiring a scanning stage.

\section{Acknowledgements}

The authors wish to thank the COMLAB staff and acknowledge the support from the Swiss National Fond \#20021-111841. 


\section{References}

[1] S. Ashley, “Artificial Muscles”, Scientific American, vol. 289, pp. 52-59, 2003.

[2] S. Rosset, M. Niklaus, P. Dubois, H.R. Shea, "Mechanical Characterization of a Dielectric elastomer Microactuator With Ion-Implanted Electrodes", Sensors and Actuators: A Physical (2007), doi:10.1016/j.sna.2007.12.030

[3] Carpi and D. De Rossi, "Dielectric elastomer cylindrical actuators: electromechanical modelling and experimental evaluation," Materials Science and Engineering, vol. C 24, no. 4, pp. 555-562, 2004.

[4] Pimpin, A., Suzuki, Y., and Kasagi, N., "Microelectrostrictive actuator with large out-of-plane deformation for flow-control application," Microelectromechanical Systems, Journal of 16(3), 753764 (2007).

[5] P. Dubois, S. Rosset, S. Koster, J. Stauffer, S. Mikhailov, M. Dadras, N.-F. de Rooij, and H. Shea, "Microactuators based on ion implanted dielectric electroactive polymer (EAP) membranes", Sensors and Actuators A: Physical, vol. 130-131, pp. 147-154, 2006.

[6] S. Rosset, M. Niklaus, P. Dubois, M. Dadras, and H. R. Shea, "Mechanical properties of electroactive polymer microactuators with ion implanted electrodes," EAPAD 2007. Proc. of the SPIE, 6524, pp. 10, 2007.

[7] M. Niklaus, S. Rosset, M. Dadras, P. Dubois, and H. R. Shea, "Modification of Conductivity and of Mechanical Properties of Electroactive Polymer (EAP) Thin Films by Titanium Ion Implantation," MRS 2008, Boston, USA, 2008.

[8] S. Rosset, M. Niklaus, V.Stojanov, A.Felber, P. Dubois, and H. R. Shea, "Ion implanted compliant and patternable electrodes for miniaturized dielectric elastomers acutators," EAPAD 2008, San Diego, USA, 2008.

[9] H. Lim, Y. Lee, and K. J. Kim, "Reduction in surface resistivity of polymers by plasma source ion implantation," Surface and coatings technology, vol. 160, pp. 158-164, 2002.

[10] K. Dworecki, T. Hasewaga, K. Sudlitz, and S. Wasik, "Modification of electrical properties of polymer membranes by ion implantation," Nuclear instruments and methods in physics research B, vol. 166-167, pp. 646-649, 2000.

[11] Y. Wu, T. Zhang, and G. Zhou, "Electrical properties of polymer modified by metal ion implantation," Nuclear instruments and methods in physics research, vol. B 169, pp. 89-93, 2000.

[12] F. Munnik, F. Benninger, S. Mikhailov, A. Bertsch, P. Renaud. T. Asmus and G. K. Wolf, "Modification and structuring of conducting polymer films on insulating substrates by ion beam treatment," Nuclear instruments and methods in physics research B, vol. 166-167, pp. 732-736, 2000 .

[13] S. Rosset, M. Niklaus, P. Dubois, and H. R. Shea, "Performance characterization of miniaturized dielectric elastomers fabricated using metal ions implantation," proc. of MEMS 2008, Tucson, USA, pp.503-506, 2008.

[14] SRIM: SRIM - The Stopping and Range of Ions in Mattersoftware at: http://www.srim.org/ 